A LETTERS JOURNAL EXPLORING THE FRONTIERS OF PHYSICS

\title{
OFFPRINT
}

\section{Quantitative relations between risk, return and firm size}

B. Podobnik, D. Horvatic, A. M. Petersen and H. E. Stanley EPL, 85 (2009) 50003

Please visit the new website www.epljournal.org 


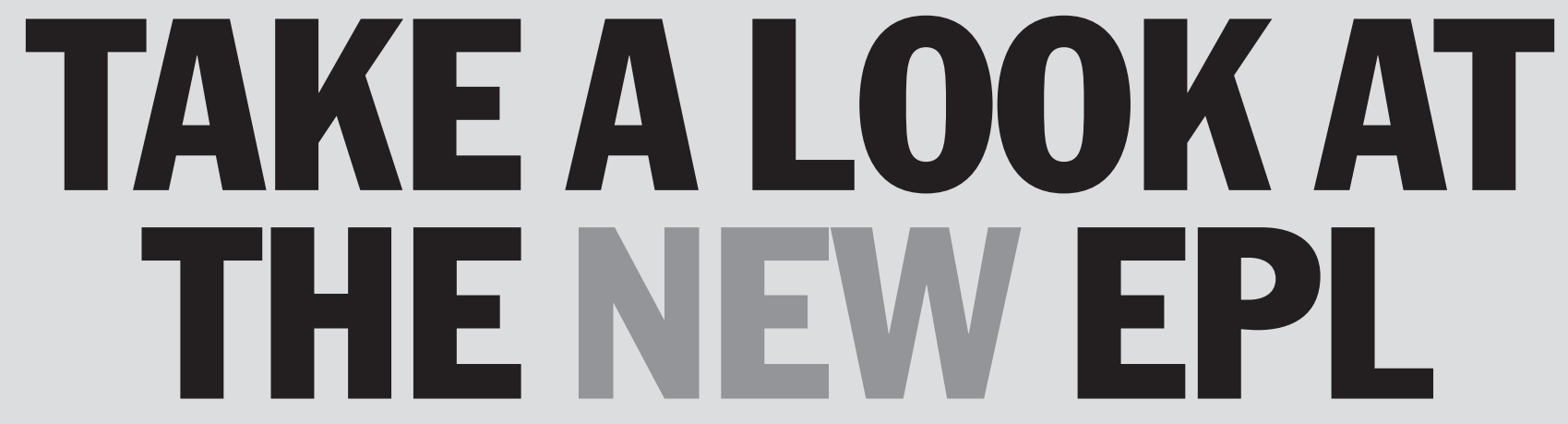

\section{Europhysics Letters (EPL) has a new online home at www.epljournal.org}

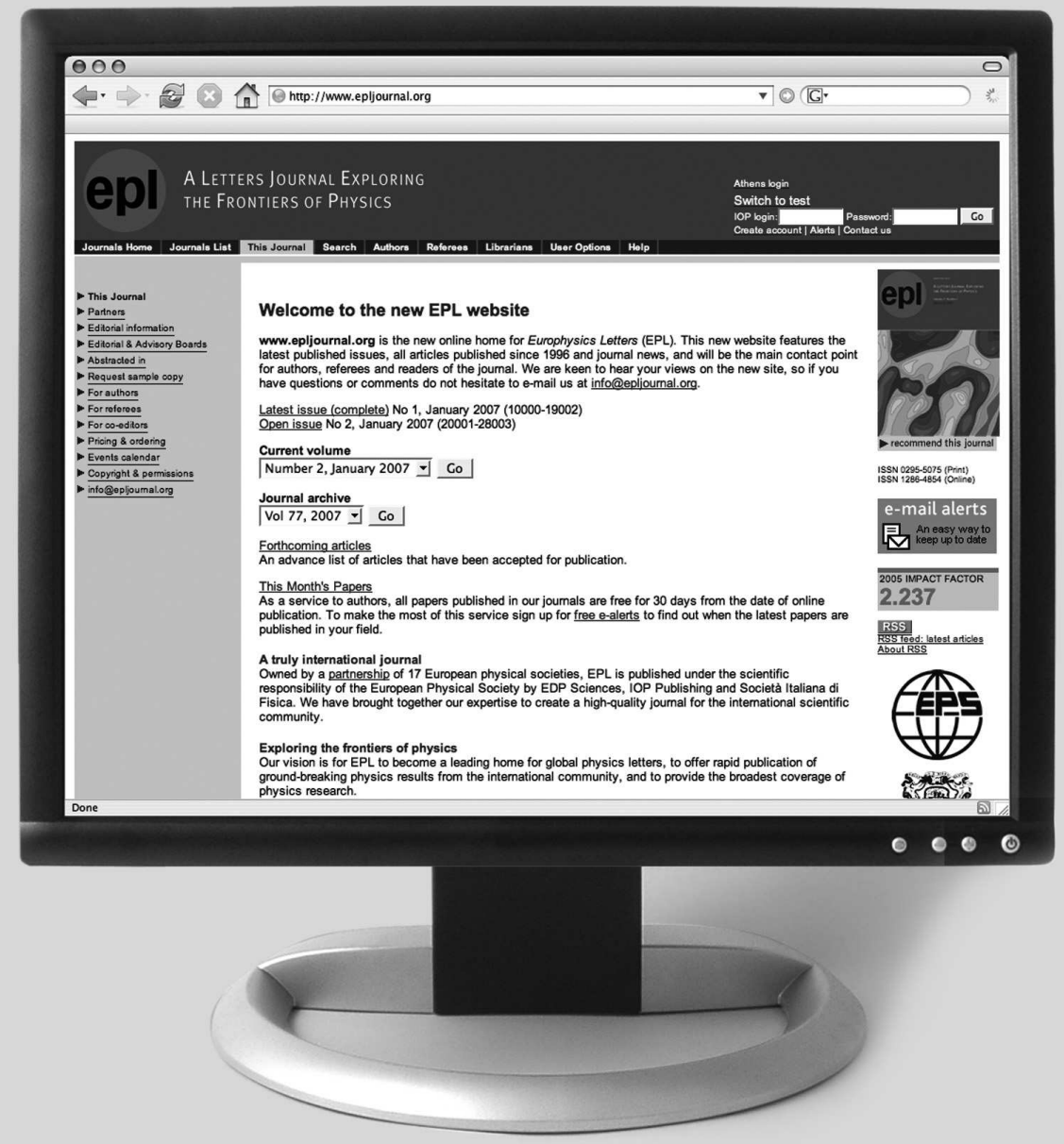

Take a look for the latest journal news and information on:

- reading the latest articles, free!

- receiving free e-mail alerts

- submitting your work to EPL 


\title{
Quantitative relations between risk, return and firm size
}

\author{
B. Podobnik ${ }^{1,2,3(a)}$, D. Horvatic ${ }^{4}$, A. M. Petersen ${ }^{1}$ and H. E. Stanley ${ }^{1}$ \\ ${ }^{1}$ Center for Polymer Studies and Department of Physics, Boston University - Boston, MA 02215, USA \\ 2 Department of Physics, Faculty of Civil Engineering, University of Rijeka - 51000 Rijeka, Croatia \\ ${ }^{3}$ Zagreb School of Economics and Management - 10000 Zagreb, Croatia \\ ${ }^{4}$ Department of Physics, University of Zagreb - 10000 Zagreb, Croatia
}

received 28 October 2008; accepted in final form 9 February 2009

published online 18 March 2009

PACS 02.50.Ey - Stochastic processes

PACS 05.40.-a - Fluctuation phenomena, random processes, noise, and Brownian motion

PACS $89.90 .+n-$ Other topics in areas of applied and interdisciplinary physics

\begin{abstract}
We analyze - for a large set of stocks comprising four financial indices - the annual logarithmic growth rate $R$ and the firm size, quantified by the market capitalization $M C$. For the Nasdaq Composite and the New York Stock Exchange Composite we find that the probability density functions of growth rates are Laplace ones in the broad central region, where the standard deviation $\sigma(R)$, as a measure of risk, decreases with the $M C$ as a power law $\sigma(R) \sim(M C)^{-\beta}$. For both the Nasdaq Composite and the S\&P 500, we find that the average growth rate $\langle R\rangle$ decreases faster than $\sigma(R)$ with $M C$, implying that the return-to-risk ratio $\langle R\rangle / \sigma(R)$ also decreases with $M C$. For the S\&P 500, $\langle R\rangle$ and $\langle R\rangle / \sigma(R)$ also follow power laws. For a 20-year time horizon, for the Nasdaq Composite we find that $\sigma(R)$ vs. $M C$ exhibits a functional form called a volatility smile, while for the NYSE Composite, we find power law stability between $\sigma(r)$ and $M C$.
\end{abstract}

Copyright (C) EPLA, 2009

Estimations of risk and return are of interest in both finance and physics [1-12]. Both risk and return (growth rate) generally decrease with increasing firm size $[4,10,12]$, but what are the functional dependences? Does risk or return decay faster? The distribution of returns is nonGaussian, but what is the functional dependence? To address these questions, we quantify the relationship between the average growth rate of stock price and firm size, between risk and firm size, and between return-to-risk ratio and firm size.

We analyze Bloomberg data on the stocks comprising four common stock indices, the New York Stock Exchange (NYSE) Composite Index, the S\&P 500, the Nasdaq Composite Index, and the FTSE All-Share Index [13]. For each stock we know the stock price and market capitalization $(M C)$ for each year, where $M C$ is calculated as the number of stocks outstanding multiplied by the price of the stock. We define an annual logarithmic growth rate $R_{t} \equiv \ln \left(S_{t} / S_{t-1}\right)$, where $S_{t}$ and $S_{t-1}$ are the stock prices in two consecutive years. We do not mix data from different market indices, in contrast to some other investigations [4], which enables us to investigate possible differences among these markets.

\footnotetext{
(a) E-mail: bp@phy.hr
}

Previous studies found that the broad central region of the probability density function (pdf) of returns exhibits slow convergence over a time scale $\Delta t$ from a truncated stable Levy distribution ( $\Delta t<30$ minutes) to a Gaussian distribution, in agreement with the central-limit theorem $[1,14,15]$. Here we test this convergence, finding that on the 1-year time scale, the pdf of returns $R$ assumes a form that is between the stable Levy and the Gaussian distributions. The form of the pdf for annual returns $R$ is important, since in practice, financial contracts are commonly based on times scales that are greater than 3 months into the future. We first analyze 6679 stocks comprising the Nasdaq Composite Index covering the 6-year time horizon, January 1, 2002 to January 1, 2008 (for which the index content is available). In order to assess the common properties of the market, we aggregate all 11836 pairs $\left(R_{t}, M C_{t}\right)$ into one common data set. In fig. 1(a) we show $P(R)$ for two different ranges of $M C$ $\left(M C<7.8 \times 10^{7}\right.$ and $\left.M C>8.4 \times 10^{9}\right)$. Each $P(R)$ displays

a) an approximately double-exponential (Laplace) form,

$$
P(R)=\frac{1}{\sqrt{2} \sigma(R)} e^{-(\sqrt{2}|R-\langle R\rangle| / \sigma(R))},
$$

b) the standard deviation $\sigma(R)$ depends on the $M C$, where smaller $\sigma(R)$ corresponds to larger $M C$ values. 

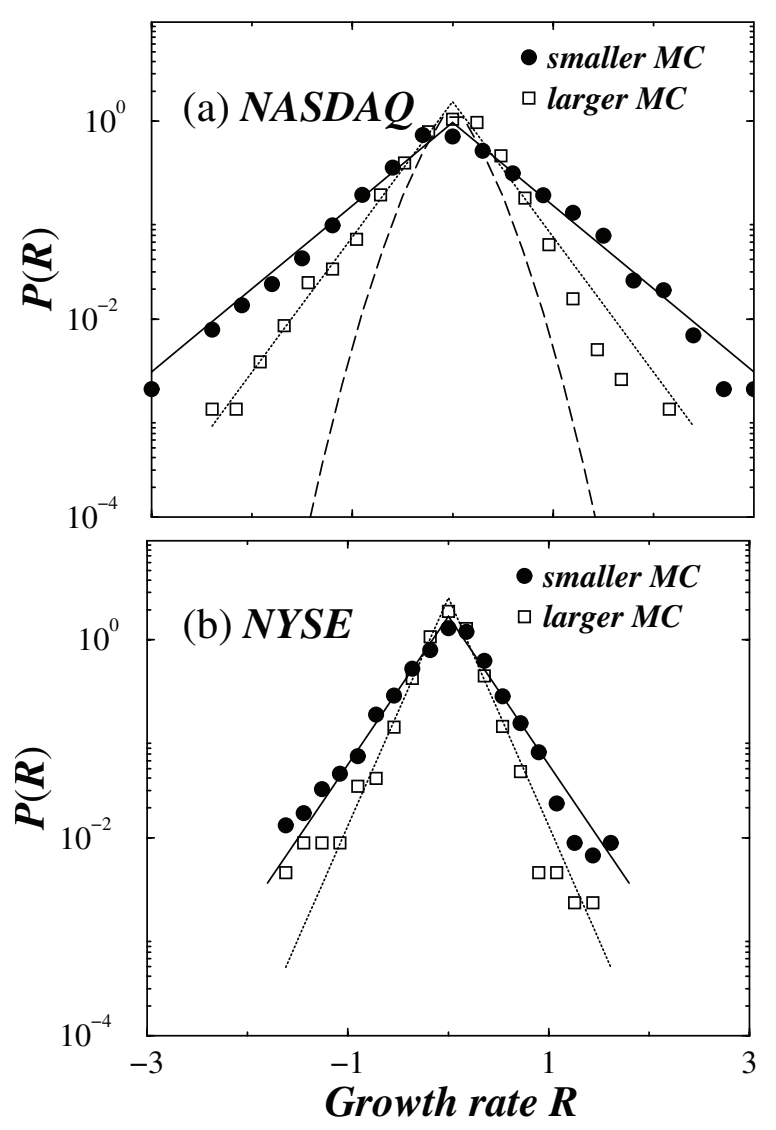

Fig. 1: The pdf $P\left(R_{t}\right)$ of annual growth rates $R_{t} \equiv \ln \left(S_{t} / S_{t-1}\right)$ of stock prices $S_{t}$ as a function of market capitalization $(M C)$. (a) Stocks comprising the Nasdaq Composite Index. For the 6 -year time horizon from $1 / 1 / 2002$ to $1 / 1 / 2008$, there are $11836 R_{t}$ values. We subdivide the 11836 pairs $(R, M C)$ into three groups of equal size, with $M C$ values smaller than $7.8 \times$ $10^{7}$, with $M C$ values $7.8 \times 10^{7}<M C<8.4 \times 10^{9}$, and $M C$ values larger than $8.4 \times 10^{9}$. Shown are two pdfs $P(R)$ where the standard deviation $\sigma(R)$ depends on $M C$. For comparison we plot a Gaussian pdf (dashed parabola) with identical $\sigma(R)$ as the pdf for the largest $M C$ values. (b) Stocks comprising the New York Stock Exchange Composite Index for the 5 -year period from $1 / 1 / 2003$ to $1 / 1 / 2008$. We subdivide the 7524 pairs $(R, M C)$ into three groups, with $M C$ values smaller than $1.3 \times 10^{7}$, with $M C$ values $1.3 \times 10^{7}<M C<6.9 \times 10^{9}$, and $M C$ values larger than $6.9 \times 10^{9}$. Both pdfs exhibit tent shapes, implying that $P(R)$ is an exponential function of $R$, and $\sigma(R)$ depends on $M C$.

For comparison, we also plot a Gaussian pdf (dashed parabola) in fig. 1(a) with identical $\sigma(R)$ as the Laplace pdf for the larger $M C$ values. We emphasize that our analysis is not focused on rare events (large $R$ values), which are consistent with power law distributed tails as reported in refs. [15,16]. In fig. 1(b) we observe the same properties a) and b) in our analysis of 2469 stocks comprising the NYSE Composite Index covering a 5-year period from January 1, 2003 to January 1, 2008. Note that these properties were also reported in microeconomics [17,18] and macroeconomics [19] in the analysis of firm growth rates and country growth rates, respectively.

To quantitatively investigate how $\sigma(R)$ depends on $M C$, we bin the data for the Nasdaq Composite Index into ten equal subintervals of $\log (M C)$. For each subinterval we calculate $\langle R\rangle$ and $\sigma(R)$. Note that in this paper we analyze the total risk and not the non-diversified risk as in refs. $[4,20]$, where the total risk refers to uncertainty measured by standard deviation, and the non-diversified risk refers to the part of the total risk which cannot be eliminated by constructing a well-diversified portfolio. In fig. 2(a) we see that $\sigma(R)$ decreases with $M C$, consistent with our qualitative observations in fig. 1(a). For the time horizon analyzed, we find that $\sigma(R)$ is well approximated, over five orders of magnitude, by the power law

$$
\sigma(R) \sim(M C)^{-\beta}
$$

with exponent and twice the standard error

$$
\beta=0.10 \pm 0.02
$$

roughly the same as the scaling exponent $\beta=0.15$ reported for the growth rates of firm size [17] and the country GDP [19]. This value of $\beta$ implies that if a firm increases its $M C$ ten times, this corresponds to a surprisingly large decrease in risk $\approx 20 \%$. Thus, larger firms have smaller standard deviations, as was shown for non-diversified risk $[4,20]$. Note that the value we obtain for $\beta$ at the $\Delta t=1$ year resolution is half the value $\beta \approx 0.2$ obtained at the $\Delta t=1$ day resolution for different empirical data [21]. As a comparison, besides the power law fit we also show the best exponential fit. Note the asymptotic deviation in the last two values of $\sigma(R)$ in fig. 2(a) from the power law fit. These two values are computed from 55 and 17 data points, respectively, and therefore we attribute the deviation from the power law fit to lack of sufficient data and finite-size effects.

Using the same binning, we also analyze the stocks comprising the NYSE Composite and the stocks comprising the S\&P500, defined as the 500 largest firms of the NYSE, over a 5-year horizon. In fig. 2(b) we find that $\sigma(R)$ corresponding to the NYSE Composite is well approximated over four orders of magnitude by a power law of eq. (2), with

$$
\beta=0.13 \pm 0.02
$$

In fig. 2(c) we repeat the analysis for the S\&P500 index, with the standard deviation $\sigma(R)$ approximated by a power law with exponent

$$
\beta=0.14 \pm 0.04
$$

One of the main topics in this paper is to find out whether risk or return decays faster, and for that reason we use a unique functional form to fit both risk and return (later in fig. 3(b)). Hence, for consistency, we fit the data 

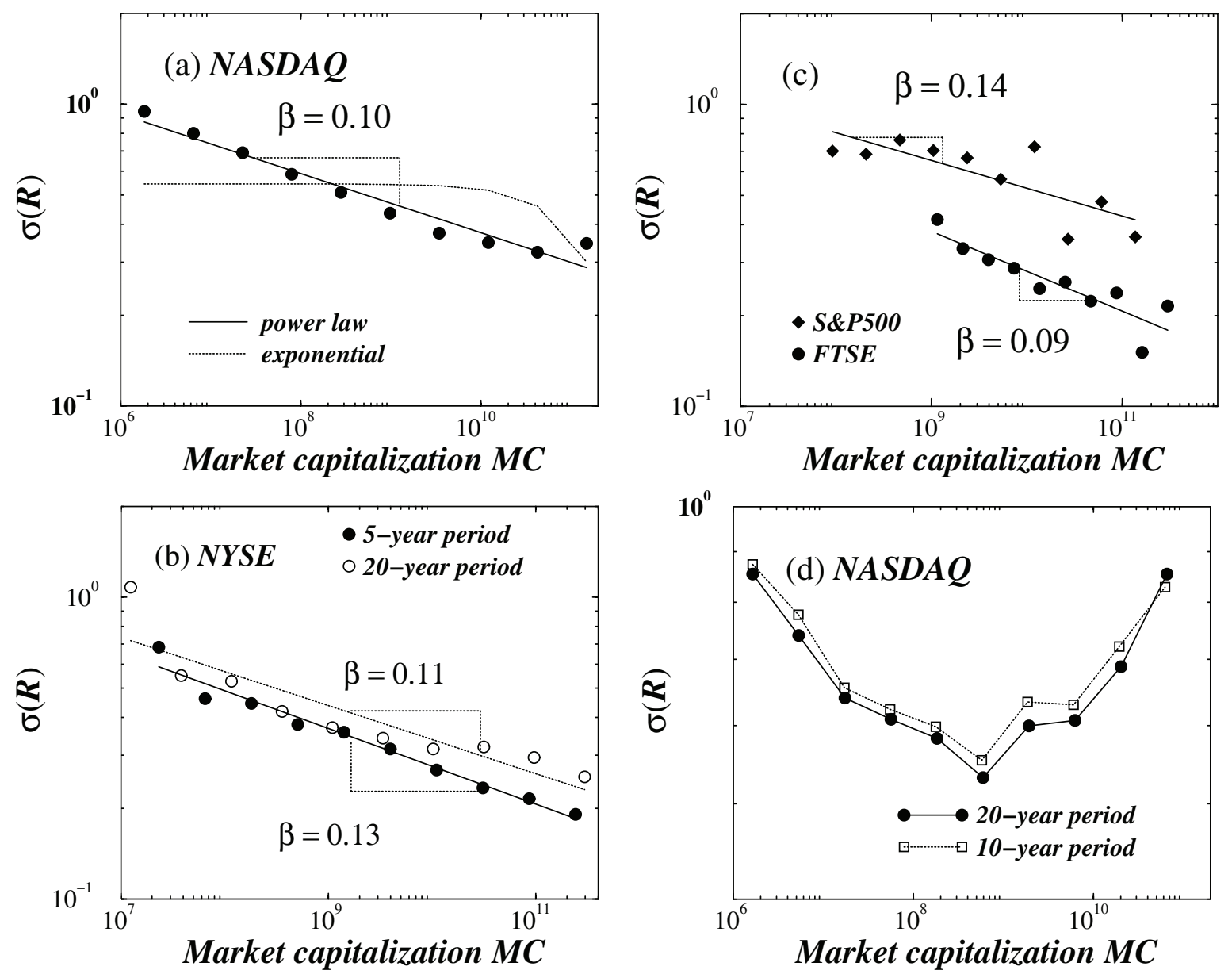

Fig. 2: Standard deviation $\sigma(R)$ of the annual growth rates $R_{t} \equiv \ln \left(S_{t} / S_{t-1}\right)$ of the stock prices $S_{t}$ as a function of the market capitalization $(M C)$. (a) Stocks comprising the Nasdaq Composite Index over the 6-year time horizon ending in 2008. The numbers of $R_{t}$ values for each of the 10 bins are: $(51,473,1496,3313,3759,1926,549,196,55,17)$. The solid line is least-square fit to the data with slope $\beta=0.10 \pm 0.02$. Clearly, the larger is the firm (larger $M C$ ), the smaller is the risk (smaller $\sigma(R)$ ). To compare, we also show the best exponential fit. (b) Stocks comprising the NYSE Composite Index in the 5 -year horizon, and the stocks over 20-year horizon included in 2007. The numbers of $R_{t}$ values for each of the 10 bins are $(11,119,514,1333,2042,1575,1064,557,253,55)$ and $(81,634,2166,4219,4745,3312,1324,460,141,48)$, respectively. Note the similarity in power law exponent for the NYSE Composite over the 5-year horizon and the 20-year horizon. Also, note the similarity in power law exponent for the NYSE Composite and the Nasdaq Composite (panel (a)) over roughly the same short horizon. (c) Stocks belonging to the S\& P500 in the 5-year horizon ending in 1/1/2008, and the FTSE All-Share Index in the 6 -year horizon ending in $1 / 1 / 2008$. We show power laws between $\sigma(R)$ and $M C$. (d) Stocks belonging to the Nasdaq Composite Index in 2007 in the most recent 20-year (10-year) horizon. We find a "volatility-smile" dependence between $\sigma(R)$ and $M C$ over the 20-year (10-year) time horizon.

in fig. 2(c) with a power law, although we agree that the presumption that all data is well approximated by power laws is not entirely satisfactory.

In order to test whether this scaling behavior is common not only to US financial markets, we analyze 988 stocks in the FTSE All-Share Index over the 6-year horizon from January 1, 2002 to January 1, 2008. In fig. 2(c) we again find power law scaling, with $\beta=0.09 \pm 0.05$.

Next we repeat the above scaling analysis for a 20 -year time horizon, using the 3039 stocks comprising the Nasdaq Composite in 2007. In contrast to the decreasing functional dependence between $\sigma(R)$ and $M C$ for the time horizon 2002-2008, we find in fig. 2(d) that for a longer 20-year time horizon, $\sigma(R)$ vs. $M C$ exhibits the behavior we may term a volatility smile. The right tail of the smile can be attributed to the crash of the technology companies and growth companies around 2000, when large companies were more volatile than small companies, opposite to what we observe in fig. 2(a). We also show that $\sigma(R)$ vs. $M C$ exhibits the same behavior over the 10-year time horizon 1998-2008. Similar volatility smiles arise in the financial theory of options [2].

In fig. 2(b) we analyze stocks comprising the NYSE Composite in 2007 over the most recent 20-year time horizon. We find that the standard deviation $\sigma(R)$ is approximated by a power law $(\beta=0.11 \pm 0.04)$, with 

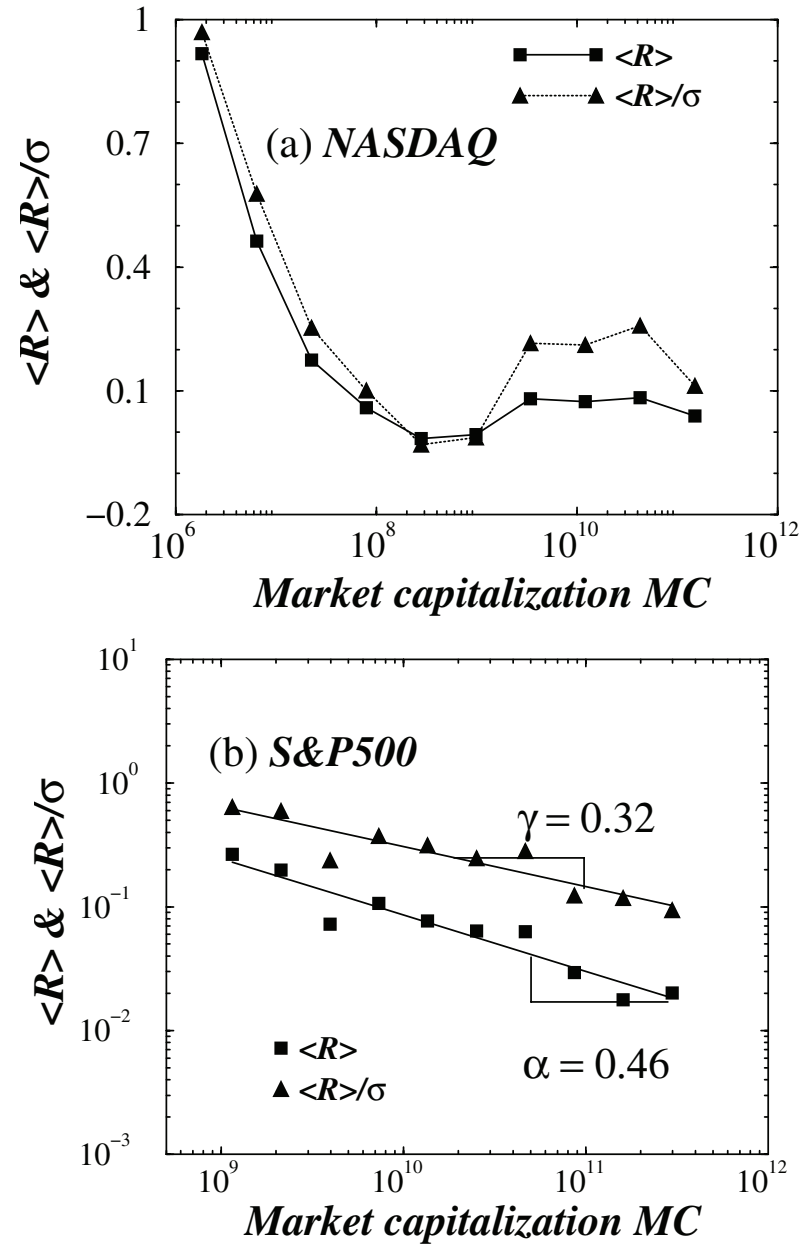

Fig. 3: Statistical properties of the annual growth rates $R_{t} \equiv$ $\ln \left(S_{t} / S_{t-1}\right)$ of the stock prices $S_{t}$ as a function of the market capitalization $(M C)$. (a) For all stocks comprising the Nasdaq Composite over the most recent 6-year time horizon (see fig. 2(a)), we show that both the average growth rate $\langle R\rangle$ and $\langle R\rangle / \sigma(R)$ decrease with increasing $M C$ up to some value of $M C$ and then gradually increase. (b) For all stocks comprising the S\& P500 over the 5-year time horizon (see fig. 2(c)), both the average growth rate $\langle R\rangle$ and $\langle R\rangle / \sigma(R)$ decrease with increasing $M C$ according to power laws.

power law exponent in agreement with that obtained for the 5 -year period $(\beta=0.13 \pm 0.02)$. This result, termed power law stability, implies that during more volatile periods, fluctuations of all stocks are increased such that the power law between $\sigma(R)$ and $M C$ does not change. It is possible that $\sigma(R)$ vs. $M C$ would be better fit by two power laws with a crossover around $M C 10^{9}$. However, we fit with a single power law so as to be consistent in our analysis of whether risk or return decays faster by fitting data to a single power law over the entire range.

Besides the graphical approach, we also apply a maximum-likelihood (ML) approach assuming the Laplace function of eq. (1) (see fig. 1), where the standard deviation has the power law form of eq. (2). For time horizons in fig. 1, we obtain the ML estimators for parameter $\beta$ which yield the maximum likelihood of generating the given samples: for Nasdaq Composite $\beta=0.13$, for NYSE Composite $\beta=0.11$, for S\&P500 $\beta=0.16$, and for FTSE All-Share Index $\beta=0.09$. These values are all in good agreement with the exponents obtained with the graphical approach (see fig. 2).

According to portfolio theory [3], an investor must take into account both $\langle R\rangle$ and $\sigma(R)$ when estimating investment performance. Sharpe introduced a single measure defined as $S_{h} \equiv\left(\langle R\rangle-R_{f}\right) / \sigma(R)[6,11]$, where $R_{f}$ is the risk-free growth rate. The larger is $S_{h}$, the better is the portfolio performance.

Next we test whether $\langle R\rangle$ or $\sigma(R)$ decreases faster with $M C$. For the Nasdaq Composite, fig. 3(a) shows that over the most recent 6 -year time horizon both $\langle R\rangle$ and $\langle R\rangle / \sigma(R)$ - a measure similar to the Sharpe ratiodecrease with $M C$ only up to a certain value of $M C$, $\approx 10^{9}$ USD, and then slowly start to increase. In fig. 3(b), the S\&P500 index shows a power law dependence $\langle R\rangle \sim(M C)^{-\alpha}$, with exponent

$$
\alpha=0.46 \pm 0.10
$$

Hence, we find that moving from small to large values of $M C$, the average growth rate $(\alpha=0.46)$ decreases even faster than the risk $(\beta=0.14$, see fig. $2(\mathrm{c}))$. Such cases, where $\alpha>\beta$, are of potential practical interest, as the inequality implies that the largest return per unit risk is found in companies with smaller $M C$. As a consequence, $\langle R\rangle / \sigma(R)$ also decreases, with $M C$ following a power law $\langle R\rangle / \sigma(R) \sim(M C)^{-\gamma}$, with exponent

$$
\gamma=0.32 \pm 0.10
$$

In conclusion, by analyzing the stocks comprising the Nasdaq Composite and NYSE Composite, we find that the growth rates follow Laplace distributions in the broad central region with a standard deviation $\sigma(R)$ that decreases with market capitalization $M C$ as a power law, consistent with the stochastic properties found in microeconomics and macroeconomics. For the Nasdaq Composite and S\&P500 index, we showed that the average growth rate decreases faster than risk with $M C$, implying that the average return-to-risk ratio decreases with $M C$. Our results are potentially valuable: in the approach where both average growth rate and risk are considered, a famous statement "All the eggs should not be placed into the same basket" can now be extended to: "Better choose baskets with smaller eggs."

We thank J. Cvitanic, X. Gabaix and M. A. SALINGER for valuable discussions and the Ministry of Science of Croatia and the NSF for financial support. 


\section{REFERENCES}

[1] TAkayasu H. (Editor), Empirical Science of Financial Fluctuations: The Advent of Econophysics (Springer, Berlin) 2002.

[2] Hull J. C., Options, Futures and Other Derivatives (Prentice Hall, New York) 2000.

[3] Markowitz H. M., J. Finance, 7 (1952) 77.

[4] Fama E. F. and French K. R., J. Finance, 47 (1992) 427.

[5] Sharpe W. F., J. Finance, 19 (1964) 425.

[6] Sharpe W. F., J. Bus., 39 (1966) 119.

[7] Treynor J. L. and Black F., J. Bus., 46 (1973) 66.

[8] Merton R. C., J. Financ. Econ., 8 (1980) 323.

[9] French K. R., Schwert G. W. and Stambaugh R. F., J. Financ. Econ., 19 (1987) 3.
[10] Fama E. F. and French K. R., J. Financ. Econ., 33 (1993) 3.

[11] Sharpe W. F., Journal of Portf. Manage., 21 (1994) 49.

[12] Davis J. L., FAma E. F. and French K. R., J. Finance, 55 (2000) 389.

[13] The data are available at Bloomberg: http://www. bloomberg.com.

[14] Mantegna R. N. and Stanley H. E., Nature, 376 (1995) 46.

[15] Gopikrishnan P. et al., Phys. Rev. E, 60 (1999) 5305.

[16] Lux T., Appl. Financ. Econ., 6 (1996) 463.

[17] Stanley M. H. R. et al., Nature, 379 (1996) 804.

[18] Amaral L. A. N. et al., J. Phys. I, 7 (1997) 621.

[19] Lee Y. et al., Phys. Rev. Lett., 81 (1998) 3275.

[20] Chan K. C. and Chen Nai-Fu., J. Finance, 63 (1988) 309.

[21] Plerou V. et al., Phys. Rev. E, 60 (1999) 6519. 